\title{
LEVEL CROSSING ALONG SPHALERON BARRIERS
}

\author{
Jutta Kunz \\ Fachbereich Physik, Universität Oldenburg, Postfach 2503 \\ D-26111 Oldenburg, Germany \\ and \\ Instituut voor Theoretische Fysica, Rijksuniversiteit te Utrecht \\ NL-3508 TA Utrecht, The Netherlands \\ Yves Brihaye \\ Faculté des Sciences, Université de Mons-Hainaut \\ B-7000 Mons, Belgium
}

March 1, 1994

\begin{abstract}
In the electroweak sector of the standard model topologically inequivalent vacua are separated by finite energy barriers, whose height is given by the sphaleron. For large values of the Higgs mass there exist several sphaleron solutions and the barriers are no longer symmetric. We construct paths of classical configurations from one vacuum to a neighbouring one and solve the fermion equations in the background field configurations along such paths, choosing the fermions of a doublet degenerate in mass. As in the case of light Higgs masses we observe the level crossing phenomenon also for large Higgs masses.
\end{abstract}




\section{Introduction}

In 1976 't Hooft [1] observed that the standard model does not absolutely conserve baryon and lepton number due to the Adler-Bell-Jackiw anomaly. In particular 't Hooft considered spontaneous fermion number violation due to instanton induced transitions, finding a negligible amplitude for such processes. At high energies, however, fermion number violating tunnelling transitions between topologically distinct vacua might become observable at future accelerators [2,3].

Manton [4] considered the possibility of fermion number violation in the standard model from another point of view. Investigating the topological structure of the configuration space of the Weinberg-Salam theory, Manton showed that there are noncontractible loops in configuration space, and predicted the existence of a static, unstable solution of the field equations, a sphaleron with Chern-Simons charge $N_{\mathrm{CS}}=1 / 2$ [5], representing the top of the energy barrier between topologically distinct vacua.

At finite temperature this energy barrier between topologically distinct vacua can be overcome due to thermal fluctuations of the fields, and fermion number violating vacuum to vacuum transitions involving changes of baryon and lepton number can occur. Baryon number violation in the standard model due to thermal transitions over the barrier may be relevant for the generation of the baryon asymmetry of the universe [6-10].

The change of fermion number during a vacuum to vacuum transition over the barrier is associated with the level crossing of one fermionic mode. A fermionic eigenstate emerges from the continuum of positive energy states, crosses zero energy and dives into the Dirac sea of negative energy states.

While it has been known since some time, that the fermions possess a zero mode in the background field of the sphaleron [11-13], the level crossing phenomenon in the background field of the sphaleron barrier was only demonstrated recently, for fermion doublets degenerate in mass, moderate values of the Higgs mass and vanishing mixing angle [14-16].

For large values of the Higgs mass the barrier separating topologically distinct vacua becomes asymmetric, the top of the barrier being now represented by a bisphaleron with Chern-Simons charge $N_{\mathrm{CS}} \neq 1 / 2[17,18]$. Here we demonstrate the level crossing phenomenon for fermions in the background field of such asymmetric barriers for large values of the Higgs mass. We numerically determine the fermion eigenvalues along various paths from one vacuum to another, passing a bisphaleron. In particular we consider the extremal energy path [19], which however becomes unsatisfactory for large values of the Higgs mass [20], and construct improved paths. We again assume that the fermions of a doublet are degenerate in mass. This assumption, violated in the standard model, allows for spherically symmetric ansätze for all of the fields, when the mixing angle dependence is neglected (which is an excellent approximation [21,22]). 
We briefly review in section 2 the Weinberg-Salam Lagrangian with the approximations employed. In section 3 we discuss paths over the barrier for large values of the Higgs mass, providing the background fields for the fermions. In section 4 we derive the radial equations for the fermions and present our results for the fermion eigenvalues along the paths. We give our conclusions in section 5 .

\section{Weinberg-Salam Lagrangian}

Let us consider the bosonic sector of the Weinberg-Salam theory in the limit of vanishing mixing angle. In this limit the U(1) field decouples and can consistently be set to zero.

$$
\mathcal{L}_{\mathrm{b}}=-\frac{1}{4} F_{\mu \nu}^{a} F^{\mu \nu, a}+\left(D_{\mu} \Phi\right)^{\dagger}\left(D^{\mu} \Phi\right)-\lambda\left(\Phi^{\dagger} \Phi-\frac{v^{2}}{2}\right)^{2}
$$

with the $\mathrm{SU}(2)_{\mathrm{L}}$ field strength tensor

$$
F_{\mu \nu}^{a}=\partial_{\mu} V_{\nu}^{a}-\partial_{\nu} V_{\mu}^{a}+g \epsilon^{a b c} V_{\mu}^{b} V_{\nu}^{c},
$$

and the covariant derivative for the Higgs field

$$
D_{\mu} \Phi=\left(\partial_{\mu}-\frac{i}{2} g \tau^{a} V_{\mu}^{a}\right) \Phi
$$

The $\mathrm{SU}(2)_{\mathrm{L}}$ gauge symmetry is spontaneously broken due to the non-vanishing vacuum expectation value $v$ of the Higgs field

$$
\langle\Phi\rangle=\frac{v}{\sqrt{2}}\left(\begin{array}{l}
0 \\
1
\end{array}\right),
$$

leading to the boson masses

$$
M_{W}=M_{Z}=\frac{1}{2} g v, \quad M_{H}=v \sqrt{2 \lambda} .
$$

We employ the values $M_{W}=80 \mathrm{GeV}, g=0.65$.

For vanishing mixing angle, considering only fermion doublets degenerate in mass, the fermion Lagrangian reads

$$
\begin{aligned}
\mathcal{L}_{\mathrm{f}} & =\bar{q}_{\mathrm{L}} i \gamma^{\mu} D_{\mu} q_{\mathrm{L}}+\bar{q}_{\mathrm{R}} i \gamma^{\mu} \partial_{\mu} q_{\mathrm{R}} \\
& -f^{(q)} \bar{q}_{\mathrm{L}}\left(\tilde{\Phi} u_{\mathrm{R}}+\Phi d_{\mathrm{R}}\right)-f^{(q)}\left(\bar{d}_{\mathrm{R}} \Phi^{\dagger}+\bar{u}_{\mathrm{R}} \tilde{\Phi}^{\dagger}\right) q_{\mathrm{L}}
\end{aligned}
$$

where $q_{\mathrm{L}}$ denotes the lefthanded doublet $\left(u_{\mathrm{L}}, d_{\mathrm{L}}\right)$, while $q_{\mathrm{R}}$ abbreviates the righthanded singlets $\left(u_{\mathrm{R}}, d_{\mathrm{R}}\right)$, with covariant derivative

$$
D_{\mu} q_{\mathrm{L}}=\left(\partial_{\mu}-\frac{i}{2} g \tau^{a} V_{\mu}^{a}\right) q_{\mathrm{L}},
$$


and with $\tilde{\Phi}=i \tau_{2} \Phi^{*}$. The fermion mass is given by

$$
M_{F}=\frac{1}{\sqrt{2}} f^{(q)} v .
$$

All gauge field configurations can be classified by a charge, the Chern-Simons charge. The Chern-Simons current

$$
K_{\mu}=\frac{g^{2}}{16 \pi^{2}} \varepsilon_{\mu \nu \rho \sigma} \operatorname{Tr}\left(\mathcal{F}^{\nu \rho} \mathcal{V}^{\sigma}+\frac{2}{3} i g \mathcal{V}^{\nu} \mathcal{V}^{\rho} \mathcal{V}^{\sigma}\right)
$$

$\left(\mathcal{F}_{\nu \rho}=1 / 2 \tau^{i} F_{\nu \rho}^{i}, \mathcal{V}_{\sigma}=1 / 2 \tau^{i} V_{\sigma}^{i}\right)$ is not conserved, its divergence $\partial^{\mu} K_{\mu}$ represents the $\mathrm{U}(1)$ anomaly. The Chern-Simons charge of a configuration is given by

$$
N_{\mathrm{CS}}=\int d^{3} r K^{0}
$$

For the vacua the Chern-Simons charge is identical to the integer winding number.

\section{Classical bosonic configurations}

Topologically inequivalent vacua are separated by finite energy barriers, whose height is determined by an unstable classical solution. For small values of the Higgs mass this is the sphaleron [5], for large values of the Higgs mass the unstable solution with only one negative mode is the first bisphaleron $[17,18]$.

\subsection{Spherically symmetric ansatz}

In the limit of vanishing mixing angle the sphaleron and bisphalerons are spherically symmetric. The general static, spherically symmetric ansatz for the gauge and Higgs fields is given by [23]

$$
\begin{aligned}
\Phi & =\frac{v}{\sqrt{2}}(H(r)+i \vec{\tau} \cdot \hat{r} K(r))\left(\begin{array}{c}
0 \\
1
\end{array}\right), \\
V_{i}^{a} & =\frac{1-f_{A}(r)}{g r} \epsilon_{a i j} \hat{r}_{j}+\frac{f_{B}(r)}{g r}\left(\delta_{i a}-\hat{r}_{i} \hat{r}_{a}\right)+\frac{f_{C}(r)}{g r} \hat{r}_{i} \hat{r}_{a}, \\
V_{0}^{a} & =0,
\end{aligned}
$$

and involves the five radial functions $H(r), K(r), f_{A}(r), f_{B}(r)$ and $f_{C}(r)$.

This ansatz leads to the energy functional

$$
\begin{aligned}
E & =\frac{4 \pi M_{W}}{g^{2}} \int_{0}^{\infty} d x\left[\frac{1}{2 x^{2}}\left(f_{A}^{2}+f_{B}^{2}-1\right)^{2}+\left(f_{A}^{\prime}+\frac{f_{B} f_{C}}{x}\right)^{2}+\left(f_{B}^{\prime}-\frac{f_{A} f_{C}}{x}\right)^{2}\right. \\
& +\left(K^{2}+H^{2}\right)\left(1+f_{A}^{2}+f_{B}^{2}+\frac{f_{C}^{2}}{2}\right)+2 f_{A}\left(K^{2}-H^{2}\right)-4 f_{B} H K \\
& \left.+2 x^{2}\left(H^{\prime 2}+K^{\prime 2}\right)-2 x f_{C}\left(K^{\prime} H-K H^{\prime}\right)+\epsilon x^{2}\left(H^{2}+K^{2}-1\right)^{2}\right],
\end{aligned}
$$


where $x=M_{W} r$, the prime means derivative with respect to $x$, and

$$
\epsilon=\frac{4 \lambda}{g^{2}}=\frac{1}{2}\left(\frac{M_{H}}{M_{W}}\right)^{2}
$$

The spherically symmetric ansatz does not completely break the gauge symmetry, there is a residual invariance of the form

$$
\begin{gathered}
\left(f_{A}+i f_{B}\right) \longrightarrow e^{i \theta}\left(f_{A}+i f_{B}\right), \\
(H+i K) \longrightarrow e^{i \frac{\theta}{2}}(H+i K), \\
f_{C} \longrightarrow f_{C}+x \theta^{\prime} .
\end{gathered}
$$

In order to construct classical solutions, the residual gauge freedom has to be fixed. Here we choose the radial gauge $\left(x_{i} V_{i}=0\right)$, imposing

$$
f_{C}(x)=0
$$

This still leaves a global U(1) symmetry, which is fixed by imposing the conditions

$$
\begin{gathered}
f_{A}(0)=1, f_{B}(0)=0, \\
f_{A}(\infty)=\cos 2 \pi q, f_{B}(\infty)=\sin 2 \pi q, \\
H(\infty)=\cos \pi q, K(\infty)=\sin \pi q,
\end{gathered}
$$

and $q \in[0,1]$.

The spherically symmetric ansatz yields the Chern-Simons charge

$$
N_{\mathrm{CS}}=q+\frac{1}{2 \pi} \int_{0}^{\infty} d x\left(f_{A}^{\prime} f_{B}-f_{A} f_{B}^{\prime}\right)
$$

\subsection{Classical solutions}

In the following we denote a generic configuration of the bosonic fields by

$$
C=\left(f_{A}, f_{B}, H, K\right)
$$

We characterize a finite energy configuration further by a quadruple of numbers

$$
X(C)=\left\{H(0), q, E, N_{\mathrm{CS}}\right\} .
$$

Defining $P$ as the parity operator supplemented by a gauge transformation Eqs. (16)(18) with $\theta=2 \pi$,

$$
P\left(f_{A}, f_{B}, H, K\right)=\left(f_{A},-f_{B},-H, K\right), X(P(C))=\left\{-H(0), 1-q, E, 1-N_{\mathrm{CS}}\right\} .
$$


This choice of $P$ preserves Eqs. (20)-(22).

We are left with two (artificially) distinct vacuum configurations

$$
C_{\mathrm{vac}} \equiv V_{ \pm}=(1,0, \pm 1,0) \quad, \quad X\left(V_{+}\right)=\{1,0,0,0\} \quad, \quad X\left(V_{-}\right)=\{-1,1,0,1\} .
$$

For $\epsilon<72$ (i. e. $M_{H}<12 M_{W}$ ) only the sphaleron solution [5] is known. The sphaleron solution is invariant under the operator $P$

$$
C_{\mathrm{sp}}=\left(f_{A}, 0,0, K\right) \quad, \quad X\left(C_{\mathrm{sp}}\right)=\left\{0, \frac{1}{2}, E(\epsilon), \frac{1}{2}\right\}
$$

The non-trivial functions $f_{A}$ and $K$ are shown in Fig. 1 for several values of the Higgs mass. The classical energy increases monotonically as a function of the Higgs mass, e. g. $E(\epsilon=0) \simeq 7.23 \mathrm{TeV}, E(\epsilon=100)=11.76 \mathrm{TeV}, E(\epsilon=200)=11.99 \mathrm{TeV}$, $E(\epsilon=\infty)=12.87 \mathrm{TeV}$.

For $\epsilon>72$ further classical solutions exist $[17,18]$, the bisphalerons, for which all four functions $f_{A}, f_{B}, H$ and $K$ are non-trivial. They are shown in Fig. 2. Their energy and Chern-Simons charge both depend on $M_{H}$, e. g.

$$
\begin{aligned}
X\left(C_{\mathrm{bi}}\right)(\epsilon=100) & =\{0.53,0.53,11.69,0.563\} \\
X\left(C_{\mathrm{bi}}\right)(\epsilon=200) & =\{0.80,0.55,11.88,0.597\} \\
X\left(C_{\mathrm{bi}}\right)(\epsilon=\infty) & =\{1.00,0.56,12.06,0.625\}
\end{aligned}
$$

where the energy values $E(\epsilon)$ are given in TeV. In contrast to the sphaleron Eq. (28), the bisphalerons are not invariant under $P$ but occur as parity doublets. All bisphalerons are lower in energy than the sphaleron. Therefore the first bisphalerons represent the top of the energy barrier between inequivalent vacua for $M_{H}>12 M_{W}$.

\subsection{Unstable modes}

To obtain the unstable modes about the sphaleron and the bisphalerons, one considers

a general spherically symmetric fluctuation about the classical solutions. Insertion of the configuration

$$
\left(f_{A}, f_{B}, H, K, f_{C}\right)=\left(C_{\mathrm{cl}}, 0\right)+\left(\eta_{A}, \eta_{B}, \eta_{H}, \eta_{K}, \eta_{C}\right)
$$

into the energy functional $E$ then leads to an eigenvalue equation for the discrete modes. For $\epsilon<72$ the sphaleron has exactly one unstable mode, while for $\epsilon>72$ the sphaleron has at least two unstable modes $[18,20]$. The first bisphalerons possess only one unstable mode $[18,24]$.

The negative eigenmode gives the direction of instability. A simple calculation involving the unstable mode of the first bisphalerons therefore gives a rough impression 
of the energy barrier in the vicinity of the bisphalerons. To this end we consider the configurations

$$
\left(f_{A}, f_{B}, H, K, f_{C}\right)=\left(C_{\mathrm{bi}}, 0\right)+\lambda\left(\eta_{A}, \eta_{B}, \eta_{H}, \eta_{K}, \eta_{C}\right)
$$

where $\lambda$ is a multiplicative constant. The fluctuation eigenvector $\eta$ for the single unstable mode of the first bisphaleron is presented in Fig. 3 for $\epsilon=100$ (with the normalisation $\eta_{H}(0)=1$ ). Gauging rotating the configurations Eq. (33) into the radial gauge Eqs. (19)-(22) we find

$$
H(0)=H_{\mathrm{bi}}(0)+\lambda, \quad q(\lambda)=q_{\mathrm{bi}}-c \lambda, \quad c=\frac{1}{2 \pi} \int_{0}^{\infty} \frac{\eta_{C}}{x} d x .
$$

The configurations Eq. (33) are regular and have finite energy. The energy of these configurations decreases as a function of $\lambda$ to both sides of the bisphaleron (where $\lambda=0)$ up to finite values $\lambda_{ \pm}$. For example for $\epsilon=100, c=0.085$ and

$$
\begin{aligned}
& \lambda_{-}=0.42, \quad X=\{0.95,0.492,11.56,0.518\}, \\
& \lambda_{+}=-1.00, \quad X=\{-0.47,0.615,10.87,0.666\},
\end{aligned}
$$

to be compared with the bisphaleron values $(\lambda=0)$, Eq. (29).

\subsection{Vacuum to vacuum paths}

Let us now consider vacuum to vacuum paths across the finite energy barriers. Such paths are also called non-contractible loops (NCLs) [4]. In the gauge used, a NCL represents a path in the space of finite energy configurations between the vacua $V_{+}$and $V_{-}$. Along such a path the asymptotic angle $2 \pi q$ of the functions $f_{A}$ and $f_{B}$ covers the trigonometric circle, while the boundary conditions at the origin of the functions $f_{A}$ and $f_{B}$, Eq. (20), are maintained.

The non-contractible loop constructed by Manton [4] (see also [25]) is symmetric under parity and its energy culminates at the sphaleron. It is therefore not appropriate for large Higgs masses, where the first bisphaleron represents the top of the energy barrier. A loop reaching its maximal energy at the bisphaleron was constructed by Klinkhamer [25].

A systematic technique to numerically construct an extremal path over the barrier is provided by the functional

$$
W=E+\xi \frac{8 \pi M_{W}}{g^{2}} \int d x\left(f_{A}^{\prime} f_{B}-f_{B}^{\prime} f_{A}\right)
$$

where the Chern-Simons charge is added to the energy functional by means of the Lagrange multiplier $\xi[19]$. For $\xi=0$ this functional yields the sphaleron and the 
bisphalerons, while for $\xi \rightarrow \pm 1$ the extrema of $W$ approach the vacua $V_{ \pm}$, independently of $M_{H}$ [20]. The extremal energy path obtained from $W$ is invariant under parity $P$.

For small values of the Higgs mass (up to $M_{H}<12 M_{W}$ ) the functional $W$ leads to a symmetric smooth minimal energy path over the barrier with the sphaleron at the top at $N_{\mathrm{CS}}=1 / 2$. However, for large values of the Higgs mass $\left(M_{H}>12 M_{W}\right)$ the extremal energy path obtained from the functional $W$ fails to be satisfactory in the neighborhood of the bisphaleron, due to the occurrence of a catastrophe [20]. As demonstrated in Figs. $4 \mathrm{a}$ and $4 \mathrm{~b}$, where we show the energy as a function of the Chern-Simons number along the extremal path, a spike occurs in the vicinity of the bisphaleron. The path coming from the vacuum $V_{+}$does not culminate at the bisphaleron, but increases in energy up to the sphaleron, where it continues symmetrically to the parity conjugate bisphaleron to finally reach the vacuum $V_{-}$. In Fig. 4 a we show the complete extremal energy path for $\epsilon=200$ and compare with the minimal energy path for $\epsilon=0.5$. In Fig. $4 \mathrm{~b}$ we show the energy as a function of the Chern-Simons number only in the vicinity of the bisphaleron for $\epsilon=100$. Here we additionally present the energy for the configurations Eq. (33) involving the negative mode. The plot suggests that these configurations can be used to improve the extremal path in the region of the bisphaleron.

We therefore now consider another construction of a NCL, reaching its maximal energy value at the bisphaleron. This NCL makes use of the invariance of the energy density under $P$ as well as of the unique negative eigenmode of the bisphaleron. Far enough away from the bisphaleron, the path coincides with the extremal energy path, approaching the vacua on both sides. While in the vicinity of the bisphaleron the path is altered to reach its maximal energy at the bisphaleron and to prevent the occurrence of the spike.

Let us call this path $L\left(N_{\mathrm{CS}}\right)$ and consider it in detail for $\epsilon=100$. We denote the two sets of extrema reaching the vacua $V_{+}$and $V_{-}$as $C_{\mathrm{e}}^{+}\left(N_{\mathrm{CS}}\right)$ and $C_{\mathrm{e}}^{-}\left(N_{\mathrm{CS}}\right)$, respectively. In the vicinity of the vacua $V_{ \pm}$we then choose

$$
\begin{aligned}
& L\left(N_{\mathrm{CS}}\right)=C_{\mathrm{e}}^{+}\left(N_{\mathrm{CS}}\right) \text { from } N_{\mathrm{CS}}=0 \quad \text { i.e. } X=\{1,0,0,0\} \\
& \text { to } N_{\mathrm{CS}}=0.54 \quad \text { i.e. } X=\{0.89,0.50,11.52,0.54\} \\
& L\left(N_{\mathrm{CS}}\right)=C_{\mathrm{e}}^{-}\left(N_{\mathrm{CS}}\right) \text { from } N_{\mathrm{CS}}=0.62 \text { i.e. } X=\{-0.98,0.61,9.35,0.62\} \\
& \text { to } N_{\mathrm{CS}}=1 \quad \text { i.e. } X=\{-1,1,0,1\} \text {. }
\end{aligned}
$$

In the vicinity of the bisphaleron $\left(N_{\mathrm{CS}, \mathrm{bi}}=0.563\right)$, i. e. from $N_{\mathrm{CS}}=0.54$ to 0.62 , the loop is closed by a set of suitable linear combinations of extrema of $W$ and of configurations Eq. (33), having the same asymptotic value of $q$

$$
\begin{gathered}
L\left(N_{\mathrm{CS}}\right)=(1-\beta(q)) C_{\mathrm{e}}^{\sigma}\left(N_{\mathrm{CS}}\right)+\beta(q)\left(C_{\mathrm{bi}}+\frac{q_{\mathrm{bi}}-q}{c}\left(\eta_{A}, \eta_{B}, \eta_{C}, \eta_{H}, \eta_{K}\right)\right)_{\theta(q)}, \\
q=q\left(N_{\mathrm{CS}}\right), \quad 0 \leq \beta(q) \leq 1, \quad \sigma=\operatorname{sign}\left(q_{\mathrm{bi}}-q\right)
\end{gathered}
$$


where $q$ corresponds to the asymptotic angle of the extremum $C_{\mathrm{e}}^{\sigma}\left(N_{\mathrm{CS}}\right)$ (the coefficient in front of $\eta$ is chosen accordingly (see Eq. (33)), and the subscript $\theta(q)$ denotes that the configurations are gauge rotated to the radial gauge. Note, that it is essential to combine configurations with identical asymptotic angle $q$ in order to keep the energy finite. The configurations Eqs. (42)-(43) have to coincide with the extremal configurations Eqs. (39) and (40), when $N_{\mathrm{CS}}=0.54$ and 0.62 , respectively, and with the bisphaleron for $N_{\mathrm{CS}}=0.563$. Therefore the function $\beta(q)$ has to obey the conditions

$$
\begin{aligned}
\beta\left(q_{\mathrm{bi}}\right) & =1, \quad q_{\mathrm{bi}}=0.53, \\
\beta(q=0.50) & =0, \quad \beta(q=0.61)=0 .
\end{aligned}
$$

Apart from these constraints, the function $\beta$ should increase (resp. decrease) monotonically for $q \leq q_{\mathrm{bi}}$ (resp. $q \geq q_{\mathrm{bi}}$ ). The smoothness of the NCL depends on the smoothness of $\beta$. There remains, however, a large arbitrariness in the choice of the function $\beta(q)$ as well as in the points chosen to depart from the extremal path. For a particular choice we show in Fig. 4b the energy along such a loop as a function of $N_{\mathrm{CS}}$. When departing from the left branch of the extremal path, $C_{\mathrm{e}}^{+}\left(N_{\mathrm{CS}}\right)$, the energy increases monotonically up to the bisphaleron. Beyond the bisphaleron the energy decreases monotonically, until it reaches the right branch of the extremal path, $C_{\mathrm{e}}^{-}\left(N_{\mathrm{CS}}\right)$. Thus this full vacuum to vacuuum path is smooth and asymmetric, culminating at the bisphaleron. Of course, the parity conjugate path passes through the parity conjugate bisphaleron.

Somewhere along the NCL the Higgs field must vanish at the origin [4,19]. Considering the complete path Eqs. (38)-(43), this happens at a point of the part Eqs. (42)-(43) of the path. There the function $H(x)$ changes sign at the origin. (In contrast to the loops of Refs. $[4,19]$ the function $H(x)$ remains non-trivial along the whole path.)

\section{Fermions}

We now derive and solve the fermion equations in the background field configurations of the vacuum to vacuum paths considered above. To retain spherical symmetry we consider only fermion doublets degenerate in mass.

\subsection{Fermion equations}

From the fermion Lagrangian (6) we obtain the eigenvalue equations for the lefthanded doublet

$$
i D_{0} q_{\mathrm{L}}+i \sigma^{i} D_{i} q_{\mathrm{L}}-f^{(q)}\left(\tilde{\Phi} u_{\mathrm{R}}+\Phi d_{\mathrm{R}}\right)=0,
$$

and for the righthanded singlets

$$
i \partial_{0}\left(\begin{array}{c}
u_{\mathrm{R}} \\
d_{\mathrm{R}}
\end{array}\right)-i \sigma^{i} \partial_{i}\left(\begin{array}{c}
u_{\mathrm{R}} \\
d_{\mathrm{R}}
\end{array}\right)-f^{(q)}\left(\begin{array}{c}
\tilde{\Phi}^{\dagger} q_{\mathrm{L}} \\
\Phi^{\dagger} q_{\mathrm{L}}
\end{array}\right)=0 .
$$


We employ the spherically symmetric ansatz for the fermion eigenstates, the hedgehog ansatz,

$$
\begin{aligned}
q_{\mathrm{L}}(\vec{r}, t) & =e^{-i \omega t}\left(G_{\mathrm{L}}(r)+i \vec{\sigma} \cdot \hat{r} F_{\mathrm{L}}(r)\right) \chi_{\mathrm{h}}, \\
q_{\mathrm{R}}(\vec{r}, t) & =e^{-i \omega t}\left(G_{\mathrm{R}}(r)+i \vec{\sigma} \cdot \hat{r} F_{\mathrm{R}}(r)\right) \chi_{\mathrm{h}},
\end{aligned}
$$

with the hedgehog spinor satisfying the spin-isospin relation $\vec{\sigma} \chi_{\mathrm{h}}+\vec{\tau} \chi_{\mathrm{h}}=0$.

The left radial functions $G_{\mathrm{L}}$ and $F_{\mathrm{L}}$ transform under the residual gauge transformation Eqs.(16)-(18) as

$$
\left(G_{\mathrm{L}}+i F_{\mathrm{L}}\right) \longrightarrow e^{-i \frac{\theta}{2}}\left(G_{\mathrm{L}}+i F_{\mathrm{L}}\right)
$$

while the right radial functions $G_{\mathrm{R}}$ and $F_{\mathrm{R}}$ are invariant.

In the radial gauge, we obtain the following set of four coupled first order differential equations [14]

$$
\begin{gathered}
\tilde{\omega} G_{\mathrm{L}}-F_{\mathrm{L}}^{\prime}-\frac{2}{x} F_{\mathrm{L}}+\frac{1-f_{A}}{x} F_{\mathrm{L}}-\frac{f_{B}}{x} G_{\mathrm{L}}-\frac{f_{C}}{2 x} G_{\mathrm{L}}-\tilde{M}_{F}\left(H G_{\mathrm{R}}+K F_{\mathrm{R}}\right)=0, \\
\tilde{\omega} F_{\mathrm{L}}+G_{\mathrm{L}}^{\prime}+\frac{1-f_{A}}{x} G_{\mathrm{L}}+\frac{f_{B}}{x} F_{\mathrm{L}}-\frac{f_{C}}{2 x} F_{\mathrm{L}}-\tilde{M}_{F}\left(H F_{\mathrm{R}}-K G_{\mathrm{R}}\right)=0 \\
\tilde{\omega} G_{\mathrm{R}}+F_{\mathrm{R}}^{\prime}+\frac{2}{x} F_{\mathrm{R}}-\tilde{M}_{F}\left(H G_{\mathrm{L}}-K F_{\mathrm{L}}\right)=0, \\
\tilde{\omega} F_{\mathrm{R}}-G_{\mathrm{R}}^{\prime}-\tilde{M}_{F}\left(H F_{\mathrm{L}}+K G_{\mathrm{L}}\right)=0,
\end{gathered}
$$

where $x$ is the dimensionless coordinate, $\tilde{\omega}$ is the dimensionless eigenvalue $\tilde{\omega}=\omega / M_{W}$ and $\tilde{M}_{F}$ is the dimensionless fermion mass $\tilde{M}_{F}=M_{F} / M_{W}$.

The eigenvalue problem Eqs. (51)-(54) for the fermions in a (bi)sphaleron-like background field requires certain boundary conditions for the fermion functions. At the origin $G_{\mathrm{L}}(x)$ and $G_{\mathrm{R}}(x)$ are finite, while $F_{\mathrm{L}}(x)$ and $F_{\mathrm{R}}(x)$ vanish, at spatial infinity all functions vanish.

\subsection{Fermionic bound states}

Previously we solved Eqs. (51)-(54) in the background field of the minimal energy sphaleron barrier for $M_{H}=M_{W}$, i. e. $\epsilon=1 / 2$ [14]. Here we extend our analysis to larger values of the Higgs mass, $M_{H}>12 M_{W}$, where the first bisphalerons are present.

For large Higgs masses the first bisphalerons represent the top of the energy barrier. Therefore we first consider the fermionic bound states in the background field of these bisphalerons. While the sphaleron always has a fermion zero mode, independent of the fermion mass and of the Higgs mass, the fermion eigenvalue in the bisphaleron background field depends on both the fermion mass and on the Higgs mass. In Fig. 5 the fermion eigenvalue $\omega$ is shown as a function of the fermion mass for several values of the 
Higgs mass. For small fermion masses we observe a critical value of the fermion mass, $M_{F}^{\text {cr }}$, below which there is no bound state. At the critical value $M_{F}^{\text {cr }}$ the fermion mode reaches the continuum, $\omega \rightarrow M_{F}$. The critical value decreases with decreasing Higgs mass and reaches zero at the first critical value of the Higgs mass, $M_{H}^{\mathrm{cr}}=12 M_{W}$, where the bisphalerons bifurcate from the sphaleron. When the Higgs mass approaches this critical value, the fermion eigenvalue approaches zero, the eigenvalue of the sphaleron for all fermion masses. In the bisphaleron background field the fermion eigenvalue is zero only at a particular value of the fermion mass. This fermion mass is in the vicinity of $115 \mathrm{GeV}$, almost independent of the Higgs mass, increasing only very slightly with increasing Higgs mass. The fermion eigenvalue in the background field of the parity conjugate bisphaleron is $-\omega$.

Let us now turn to the fermion eigenvalue along the barrier. First we consider the eigenvalue along the extremal energy path, since this path is well defined and represents part of the improved path, Eqs. (38)-(43). In Fig. 6a we present the fermion eigenvalue normalized by the fermion mass for several values of the fermion mass along the central part of the extremal path for $\epsilon=100$. The catastrophes present along the extremal path are reflected in the behaviour of the fermion eigenvalue, and there is a strong dependence on the fermion mass. At the two critical points, where the energy reaches a spike as a function of the Chern-Simons number, the fermion eigenvalue bends around, having an infinite derivative, provided that the fermion mass is large enough so that fermion bound states still exist in this region of $N_{\mathrm{CS}}$.

For light fermions bound states exist only in a narrow region around $N_{\mathrm{CS}}=1 / 2$, and we observe three distinct branches, illustrated in Fig. 6a for $M_{F}=0.8 \mathrm{GeV}$. Moving along the extremal path, a first branch starts from the positive continuum, passes zero and dives into the negative continuum. Then a second branch starts from the negative continuuum passes zero at the sphaleron and dives into the positive continuum. Finally a third branch starts from the positive continuum, passes zero and dives into the negative continuum. Thus three zero modes occur along the full extremal path, but since these modes are encountered from alternating directions, the total change in fermion number is the same as for a single level crossing.

For heavier fermions, when bound states exist also at the values of $N_{\mathrm{CS}}$, where the spikes are encountered, the fermion eigenvalue starts from the positive continuum, crosses zero, and bends backward and upward at the first spike. Then it crosses zero again at the sphaleron, reaches the second spike and bends forward and downward, to cross zero for the third time and finally reach the negative continuum. This is illustrated in Fig. $6 \mathrm{a}$ for the fermion masses $M_{F}=8 \mathrm{GeV}$ and $M_{F}=80 \mathrm{GeV}$. For heavy fermions with $M_{F}>145 \mathrm{GeV}$ the fermion eigenvalue crosses zero only once along the extremal path, at the sphaleron, as illustrated in Fig. 6 a for $M_{F}=160 \mathrm{GeV}$.

The oscillating, catastrophic behaviour of the fermion eigenvalues along the extremal path for large Higgs masses is remedied for the improved path, Eqs. (38)-(43). This is 
demonstrated in Fig. $6 \mathrm{~b}$ for a fermion mass of $M_{F}=80 \mathrm{GeV}$ and $\epsilon=100$, along the improved path presented in Fig. 4b. Here the eigenvalue decreases monotonically from the positive continuum to the negative continuum as a function of $N_{\mathrm{CS}}$, passing zero only once.

Let us now consider how the fermion eigenvalue along the improved path depends on the fermion mass. It is instructive to first inspect the critical values of the fermion mass $M_{F}^{c r}$, where the bound state enters the continuum along the extremal path, since to a large extend the extremal path coincides with the improved path, Eqs. (38)-(43). The critical values of the fermion mass are illustrated in Fig. 7 for $\epsilon=100$. While for small fermion masses fermion bound states exist only in the vicinity of $N_{\mathrm{CS}}=1 / 2$, fermion bound states exist with increasing fermion mass along increasing parts of the barrier. Reflecting again the catastophe, we observe six critical values below $M_{F}=6.8$ $\mathrm{GeV}$ and 2 critical values above $M_{F}=6.8 \mathrm{GeV}$.

It is further instructive to observe where along the extremal path the zero modes occur for various fermion masses. These fermion masses are shown in Fig. 8 for $\epsilon=100$. For zero mass fermions the three zero modes are encountered at $N_{\mathrm{CS}}=0.505, N_{\mathrm{CS}}=1 / 2$ and $N_{\mathrm{CS}}=0.495$ along the extremal path. Moving along the improved path, Eqs.(38)(43), however, only one zero mode is encountered for zero mass fermions, occurring at $N_{\mathrm{CS}}=0.505$ along the part $C_{\mathrm{e}}^{+}\left(N_{\mathrm{CS}}\right)$ of the improved path, which coincides with the extremal path.

Also for small fermion masses the fermion zero mode occurs along the extremal part $C_{\mathrm{e}}^{+}\left(N_{\mathrm{CS}}\right)$ of the improved path. Fig. 8 gives the value of the fermion mass beyond which the zero mode occurs along the intermediate part, Eqs. (42)-(43), of the improved path, depending on the point of departure of the improved path from the extremal path. For the improved path presented in Fig. 4b this fermion mass is $M_{F}=34 \mathrm{GeV}$. Since the path passes the bisphaleron, the fermion zero mode is encountered before the bisphaleron for fermions masses smaller than $M_{F}=116 \mathrm{GeV}$ and beyond the bisphaleron for fermions masses larger than $M_{F}=116 \mathrm{GeV}$. Of course the precise point along the path, where the zero mode is encountered, depends on the specific choice of the improved path, except when the zero mode occurs in the bisphaleron background field.

Analogous considerations hold for the parity conjugate path, passing the parity conjugate bisphaleron.

\section{Conclusions}

We have demonstrated the level crossing phenomenon along the energy barrier between topologically distinct vacua for large Higgs masses, when the barrier is no longer symmetric. 
We have employed various approximations in this study. To keep spherical symmetry, we have neglected the finite mixing angle and the mass splitting within the fermion doublet. While the mixing angle dependence is small [21,22], the effect of the fermion mass splitting on the fermion modes certainly needs further investigation. We have also only treated the valence fermion. The inclusion of the Dirac sea has been studied by Diakonov et al. [16] (for small Higgs masses), showing only a small effect for light fermions, but an important contribution for heavy fermions. We have further neglected the back reaction of the fermions on the barrier, which has been considered in [15] (for small Higgs masses), showing again that the effect is important only for heavy fermions, when the barrier becomes asymmetric even for small Higgs masses.

For large Higgs masses, when $M_{H}>12 M_{W}$, there are two parity conjugate barriers, and the configurations representing the top of these barriers are the two conjugate first bisphalerons. We have considered various paths over these barriers, passing a bisphaleron. The extremal path [19] is not satisfactory for these large Higgs masses, since it is parity symmetric, passing besides both bisphalerons also the sphaleron, with catastrophes in the vicinity of the bisphalerons. Therefore we have constructed an improved path involving the single negative mode of the bisphaleron. This improved path is smooth and culminates at the bisphaleron.

Solving the fermion eigenvalue equations in the background field of the bisphaleron, we observe, that the fermion eigenvalue depends on both the fermion mass and on the Higgs mass. This is in contrast to the sphaleron, which has a fermion zero mode for all fermion masses and Higgs masses. For a given Higgs mass there is only one particular value of the fermion mass, where the bisphaleron supports a zero eigenvalue. Interestingly, this fermion mass depends only very slightly on the Higgs mass.

In the background field of the extremal path the fermion eigenvalue reflects the catastrophic behaviour of the extremal path. For small fermion masses, $M_{F}<145$ $\mathrm{GeV}$, there are three zero modes encountered from alternating directions, while for larger fermion masses there is only one zero mode encountered at the sphaleron. In contrast, along the smooth improved path the fermion eigenvalue is also smooth and only a single zero mode is encountered for all fermion masses. Interestingly, this zero mode occurs almost at $N_{\mathrm{CS}}=1 / 2$, when the fermion mass tends to zero. 


\section{References}

[1] G. 't Hooft, Symmetry breaking through Bell-Jackiw Anomalies, Phys. Rev. Lett. 37 (1976) 8.

[2] A. Ringwald, High energy breakdown of perturbation theory in the electroweak instanton sector, Nucl. Phys. B330 (1990) 1.

[3] M. Mattis, and E. Mottola, eds., "Baryon Number Violation at the SSC?", World Scientific, Singapore (1990).

[4] N. S. Manton, Topology in the Weinberg-Salam theory, Phys. Rev. D28 (1983) 2019.

[5] F. R. Klinkhamer, and N. S. Manton, A saddle-point solution in the WeinbergSalam theory, Phys. Rev. D30 (1984) 2212.

[6] V. A. Kuzmin, V. A. Rubakov, and M. E. Shaposhnikov, On anomalous electroweak baryon-number non-conservation in the early universe, Phys. Lett. B155 (1985) 36.

[7] P. Arnold, and L. McLerran, Sphalerons, small fluctuations, and baryon-number violation in electroweak theory, Phys. Rev. D36 (1987) 581.

[8] P. Arnold, and L. McLerran, The sphaleron strikes back: A response to objections to the sphaleron approximation, Phys. Rev. D37 (1988) 1020.

[9] L. Carson, X. Li, L. McLerran, and R.-T. Wang, Exact computation of the smallfluctuation determinant around a sphaleron, Phys. Rev. D42 (1990) 2127.

[10] E. W. Kolb, and M. S. Turner, "The Early Universe", Addison-Wesley Publishing Company, Redwood City (1990).

[11] C. R. Nohl, Bound-state solutions of the Dirac equation in extended hadron models, Phys. Rev. D12 (1975) 1840.

[12] J. Boguta, and J. Kunz, Hadroids and sphalerons, Phys. Lett. B154 (1985) 407.

[13] A. Ringwald, Sphaleron and level crossing, Phys. Lett. B213 (1988) 61.

[14] J. Kunz and Y. Brihaye, Fermions in the background of the sphaleron barrier. Phys. Lett. B304 (1993) 141.

[15] G. Nolte and J. Kunz, Sphaleron barrier in the presence of fermions, Phys. Rev. D48 (1993) 5905. 
[16] D. Diakonov, M. Polyakov, P. Sieber, J. Schaldach and K. Goeke, Fermion sea along the sphaleron barrier, preprint RUB-TPII-62/93.

[17] J. Kunz, and Y. Brihaye, New sphalerons in the Weinberg-Salam theory, Phys. Lett. B216 (1989) 353.

[18] L. G. Yaffe, Static solutions of SU(2)-Higgs theory, Phys. Rev. D40 (1989) 3463.

[19] T. Akiba, H. Kikuchi, and T. Yanagida, Static minimum-energy path from a vacuum to a sphaleron in the Weinberg-Salam model, Phys. Rev. D38 (1988) 1937.

[20] Y. Brihaye, S. Giler, P. Kosinski, and J. Kunz, Configuration space around the sphaleron, Phys. Rev. D42 (1989) 2846.

[21] B. Kleihaus, J. Kunz, and Y. Brihaye, The electroweak sphaleron at physical mixing angle, Phys. Lett. B273 (1991) 100.

[22] J. Kunz, B. Kleihaus, and Y. Brihaye, Sphalerons at finite mixing angle, Phys. Rev. D46 (1992) 3587.

[23] R. F. Dashen, B. Hasslacher, and A. Neveu, Nonperturbative methods and extended-hadron models in field theory. III. Four-dimensional non-abelian models, Phys. Rev. D12 (1974) 4138.

[24] Y. Brihaye and J. Kunz, Normal modes around SU(2) sphalerons, Phys. Lett. B249 (1990) 90.

[25] F. R. Klinkhamer, Sphalerons, deformed sphalerons and configuration space, Phys. Lett. B236 (1990) 187. 


\section{$6 \quad$ Figure Captions}

Fig. 1:

The sphaleron functions $f_{A}(x)$ and $K(x)$ are shown for three values of the Higgs mass, $M_{H}=20 M_{W}$ (dashed), $M_{H}=\sqrt{200} M_{W}$ (solid) and $M_{H}=M_{W}$ (dotted).

Fig. 2:

The bisphaleron functions $f_{A}(x), f_{B}(x), H(x)$ and $K(x)$ are shown for two values of the Higgs mass, $M_{H}=20 M_{W}$ (dashed) and $M_{H}=\sqrt{200} M_{W}$ (solid).

Fig. 3:

The functions of the negative eigenmode of the bisphaleron $\eta_{A}(x), \eta_{B}(x), \eta_{C}(x)$, $\eta_{H}(x)$ and $\eta_{K}(x)$ are shown for two values of the Higgs mass, $M_{H}=20 M_{W}$ (dashed) and $M_{H}=\sqrt{200} M_{W}$ (solid) with the normalization $\eta_{H}(0)=1$.

Fig. 4a:

The energy $E$ (in $\mathrm{TeV}$ ) is shown as a function of the Chern-Simons number $N_{\mathrm{CS}}$ along the full extremal path from one vacuum to another for $M_{H}=20 M_{W}$ (solid) and $M_{H}=M_{W}$ (dashed).

Fig. 4b:

The energy $E$ (in $\mathrm{TeV}$ ) is shown as a function of the Chern-Simons number $N_{\mathrm{CS}}$ in the critical region of the extremal path for $M_{H}=\sqrt{200} M_{W}$ (solid). Also shown is the energy for the configurations Eq. (33) (dotted), and the energy along the improved path Eqs. (38)-(43) (dashed). The improved path and the extemal path differ only in the intermediate region Eqs. (42)-(43) but coincide at the bisphaleron. The crosses mark the bisphalerons and the plus signs the points of departure of the improved path from the extremal path.

Fig. 5:

The fermion eigenvalue $\omega$ (in $\mathrm{GeV}$ ) in the background field of the bisphaleron is shown as a function of the fermion mass $M_{F}$ (in $\mathrm{GeV}$ ) for three values of the Higgs mass, $M_{H}=20 M_{W}$ (dotted), $M_{H}=\sqrt{200} M_{W}$ (dashed) and $M_{H}=\sqrt{150} M_{W}$ (dot-dashed). Also shown are the critical value of the fermion mass $M_{F}^{\text {cr }}$, where the fermion eigenvalue reaches the continuum (long-dashed), and the zero eigenvalue of the sphaleron (solid).

Fig. 6a:

The normalized fermion eigenvalue $\omega / M_{F}$ is shown as a function of the ChernSimons number $N_{\mathrm{CS}}$ along the extremal path for the values of the fermion mass $M_{F}=$ $0.8 \mathrm{GeV}, M_{F}=8 \mathrm{GeV}, M_{F}=80 \mathrm{GeV}$ and $M_{F}=160 \mathrm{GeV}$ for $M_{H}=\sqrt{200} M_{W}$ (solid), and for comparison for $M_{F}=0.8 \mathrm{GeV}, M_{F}=8 \mathrm{GeV}$ and $M_{F}=80 \mathrm{GeV}$ for $M_{H}=M_{W}$ (dashed).

Fig. 6b:

The normalized fermion eigenvalue $\omega / M_{F}$ is shown as a function of the ChernSimons number $N_{\mathrm{CS}}$ along the critical part of the extremal path for $M_{H}=\sqrt{200} M_{W}$ for the fermion mass $M_{F}=80 \mathrm{GeV}$ (solid). Also shown is the normalized fermion 
eigenvalue $\omega / M_{F}$ along the improved path Eqs. (38)-(43) (dashed). Both paths differ only in the intermediate region Eqs. (42)-(43) and cross at the bisphaleron. The crosses mark the bisphalerons and the plus signs the points of departure of the improved path from the extremal path.

\section{Fig. 7:}

The critical fermion mass $M_{F}^{\text {cr }}$ (in $\mathrm{GeV}$ ), at which the bound state enters the continuum for a given Chern-Simons number along the extremal path, is shown as a function of the Chern-Simons number $N_{\mathrm{CS}}$ for $M_{H}=\sqrt{200} M_{W}$ (solid), and for comparison for $M_{H}=M_{W}$ (dashed).

Fig. 8:

The fermion mass $M_{F}$ (in $\mathrm{GeV}$ ), at which the zero mode occurs for a given ChernSimons number along the extremal path, is shown as a function of the Chern-Simons number $N_{\mathrm{CS}}$ for $M_{H}=\sqrt{200} M_{W}$. The vertical line represents the zero mode of the sphaleron. 


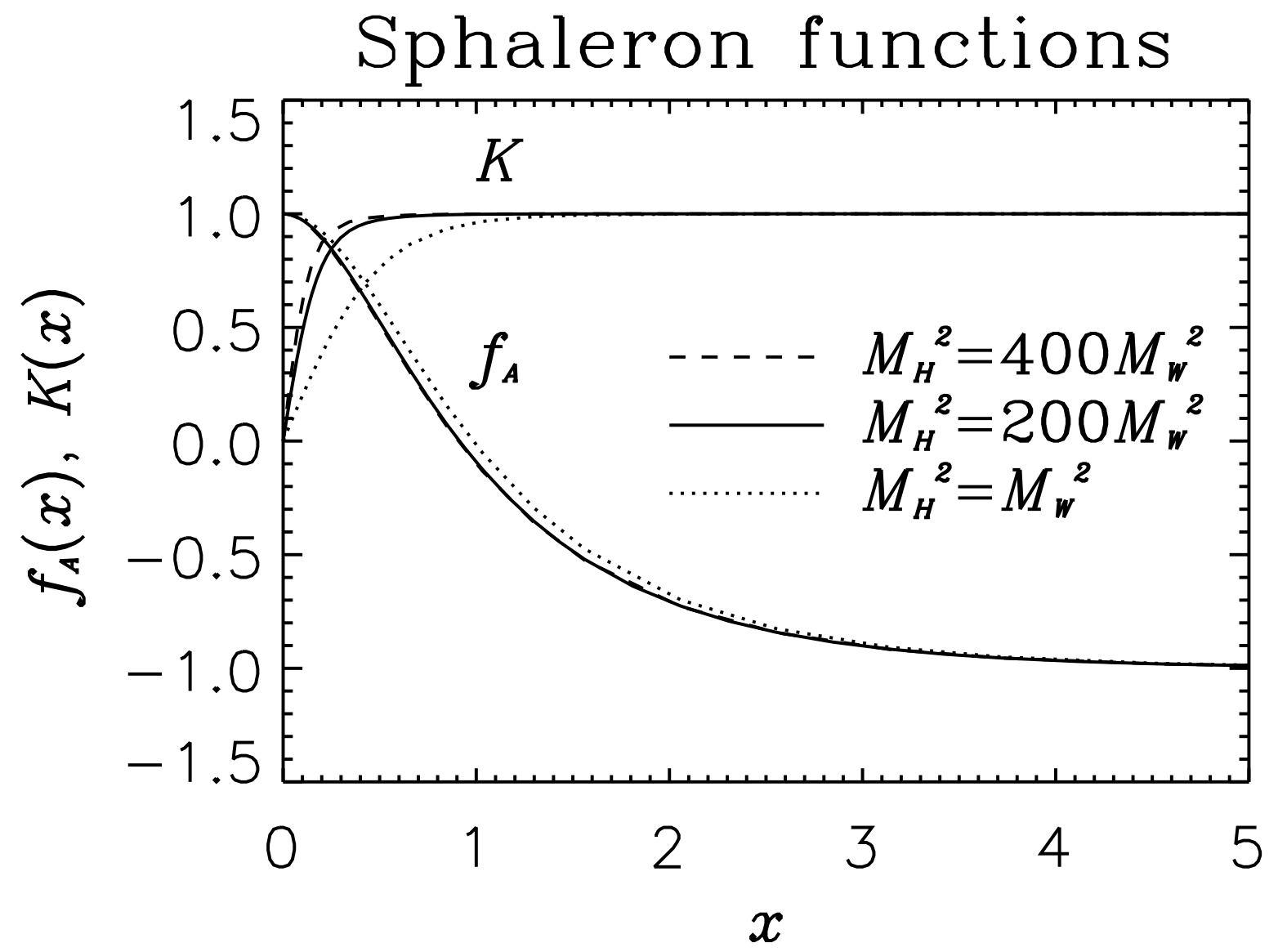


This figure "fig1-1.png" is available in "png" format from: http://arxiv.org/ps/hep-ph/9403216v1 
This figure "fig2-1.png" is available in "png" format from: http://arxiv.org/ps/hep-ph/9403216v1 


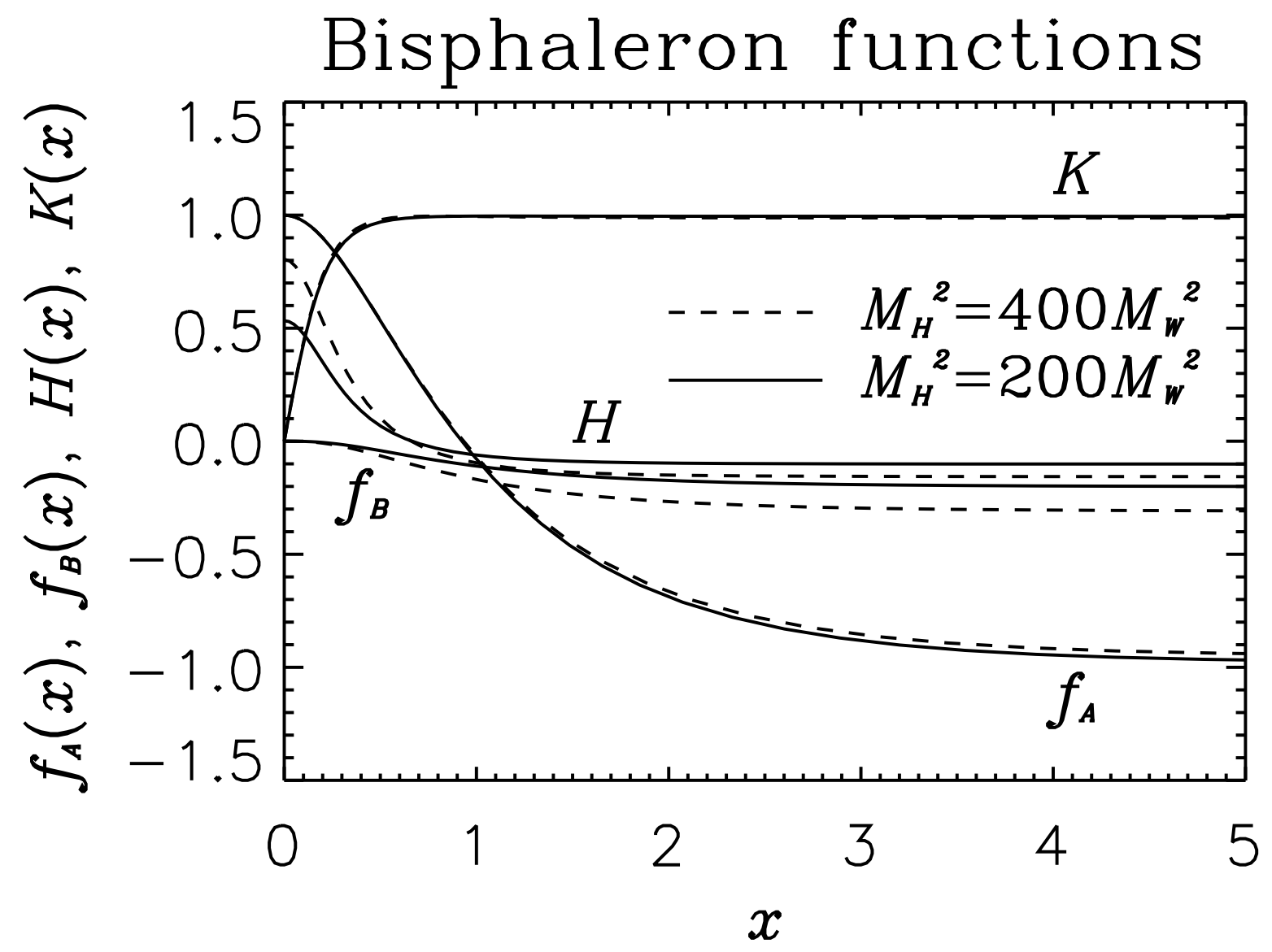


This figure "fig1-2.png" is available in "png" format from: http://arxiv.org/ps/hep-ph/9403216v1 
This figure "fig2-2.png" is available in "png" format from: http://arxiv.org/ps/hep-ph/9403216v1 


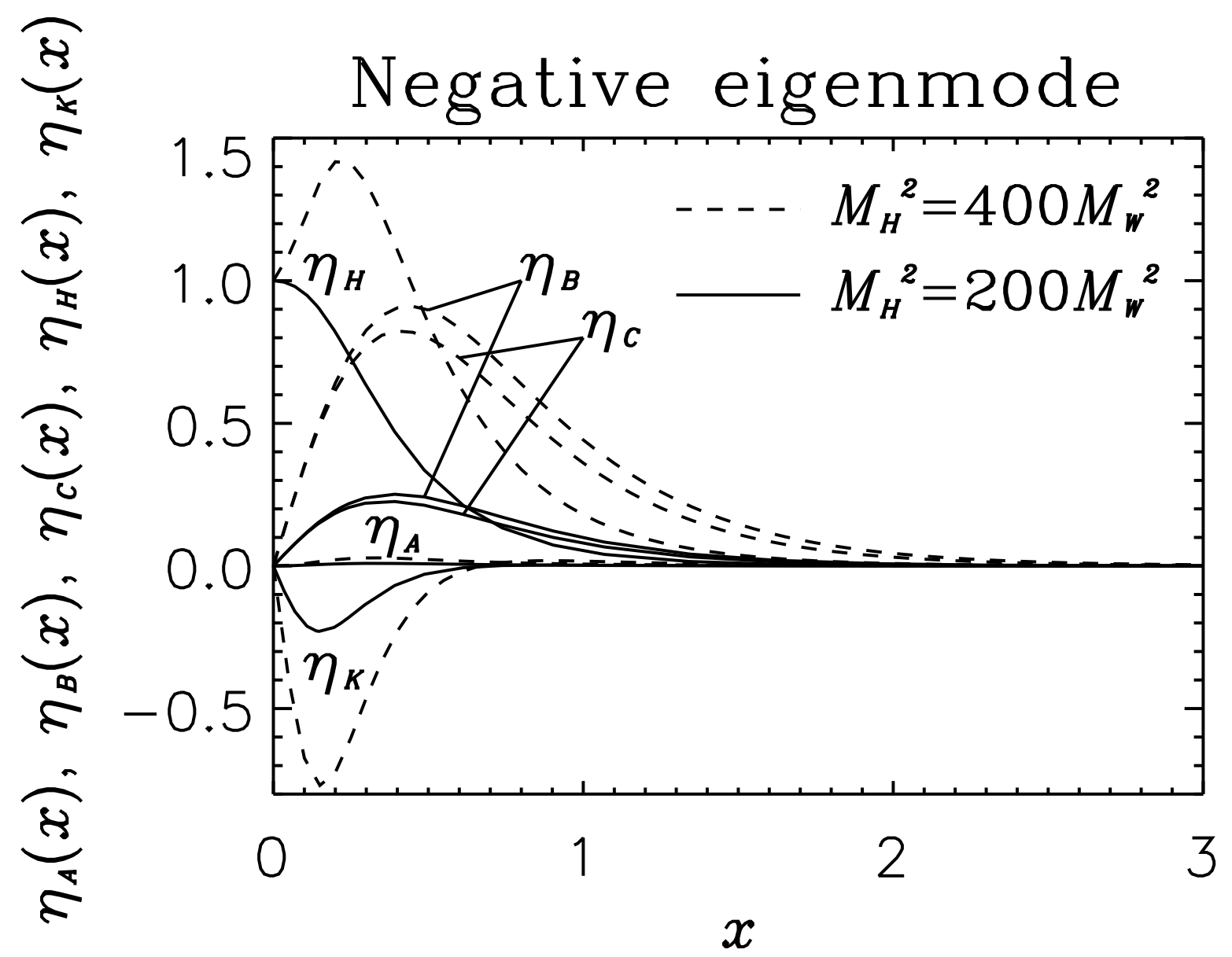


This figure "fig1-3.png" is available in "png" format from: http://arxiv.org/ps/hep-ph/9403216v1 
This figure "fig2-3.png" is available in "png" format from: http://arxiv.org/ps/hep-ph/9403216v1 
Barriers

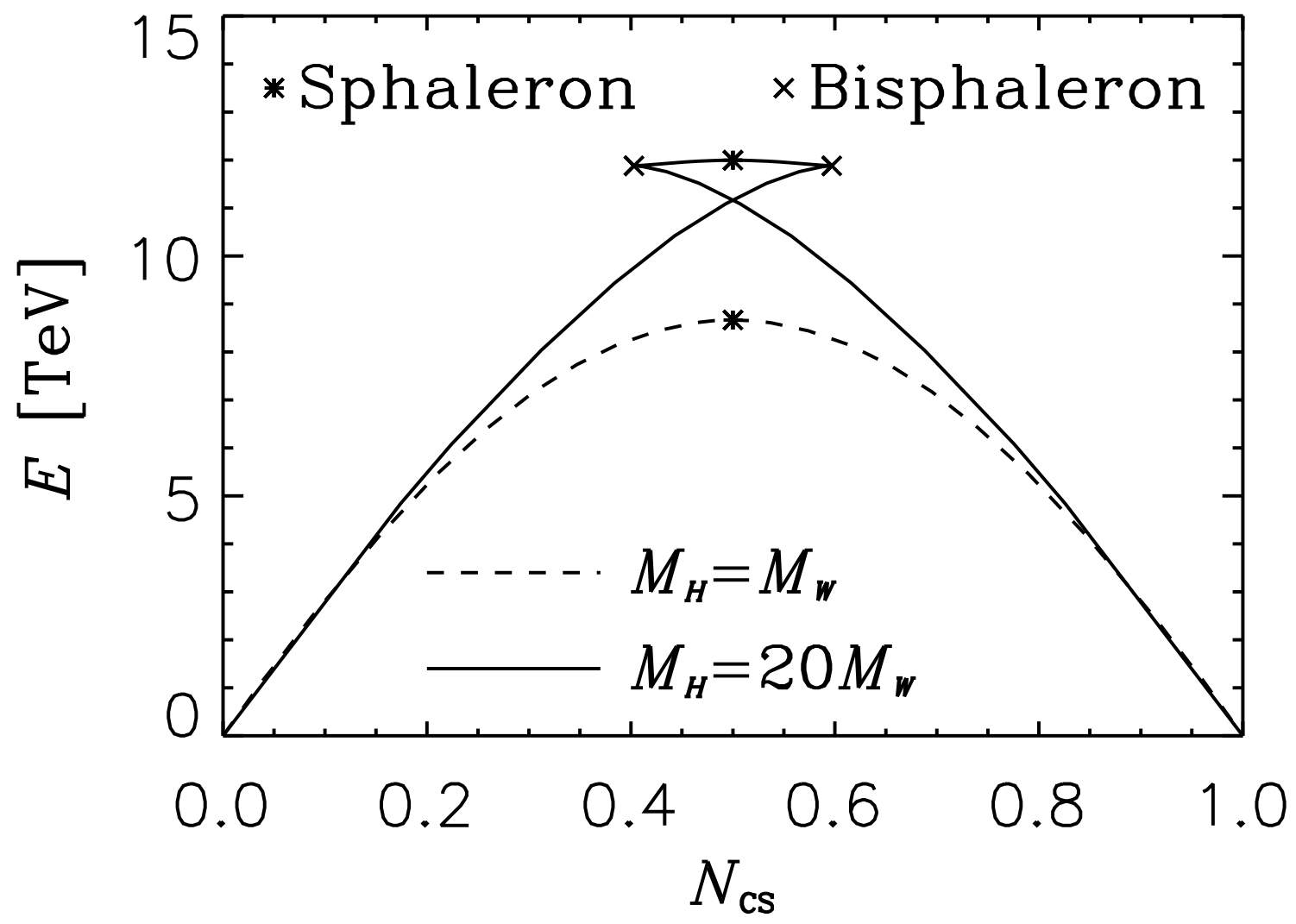




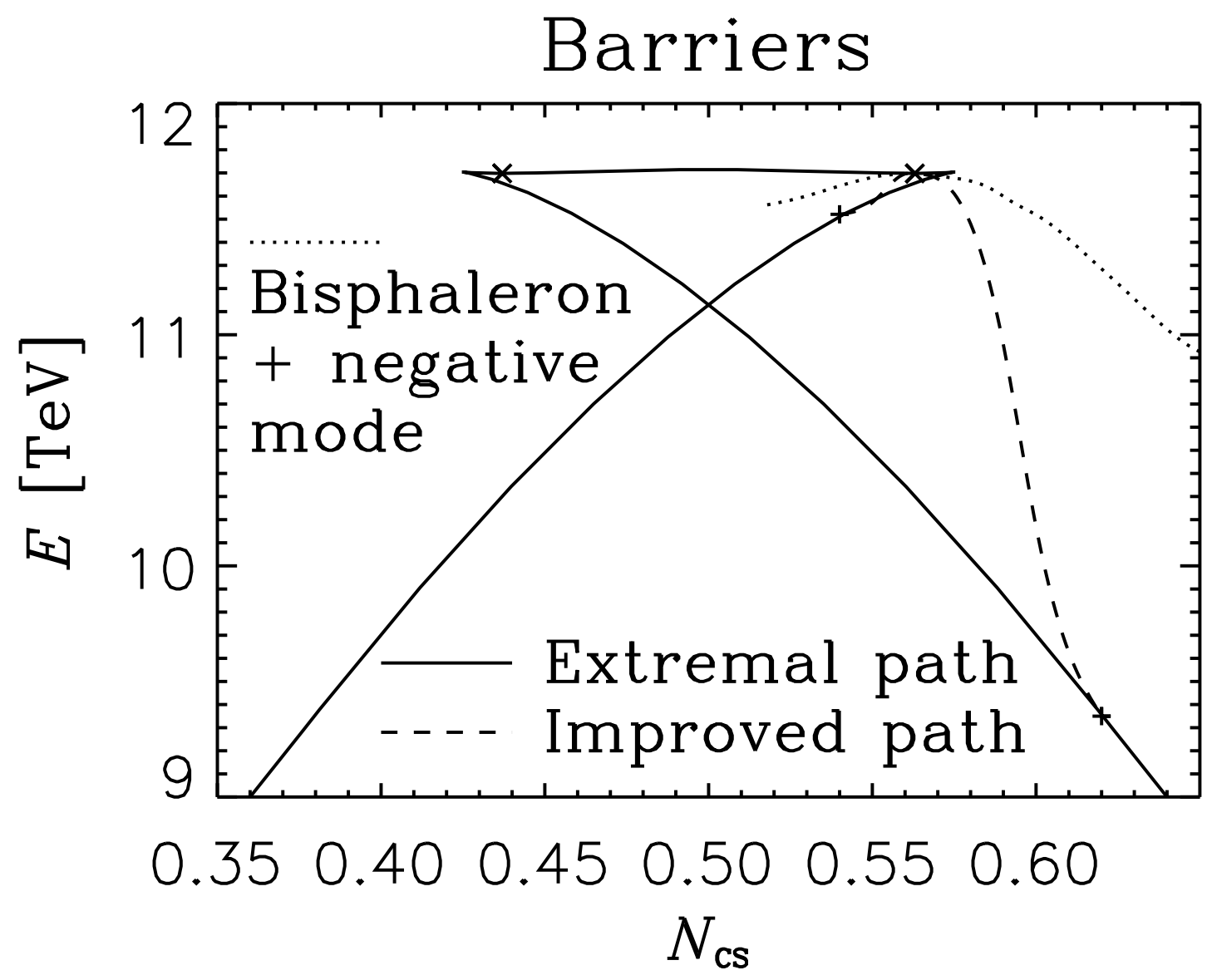


This figure "fig1-4.png" is available in "png" format from: http://arxiv.org/ps/hep-ph/9403216v1 
This figure "fig2-4.png" is available in "png" format from: http://arxiv.org/ps/hep-ph/9403216v1 


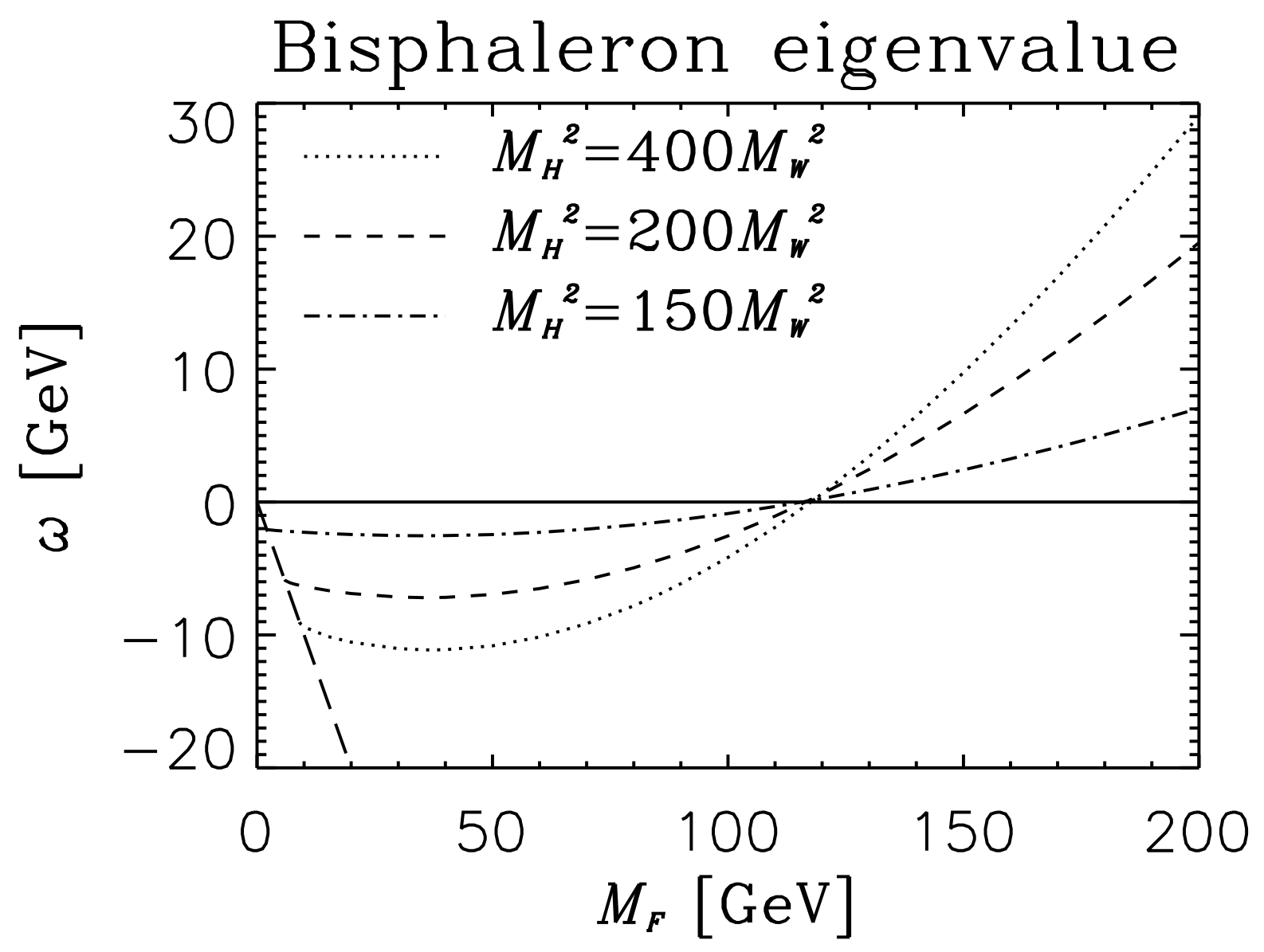


This figure "fig1-5.png" is available in "png" format from: http://arxiv.org/ps/hep-ph/9403216v1 
This figure "fig2-5.png" is available in "png" format from: http://arxiv.org/ps/hep-ph/9403216v1 


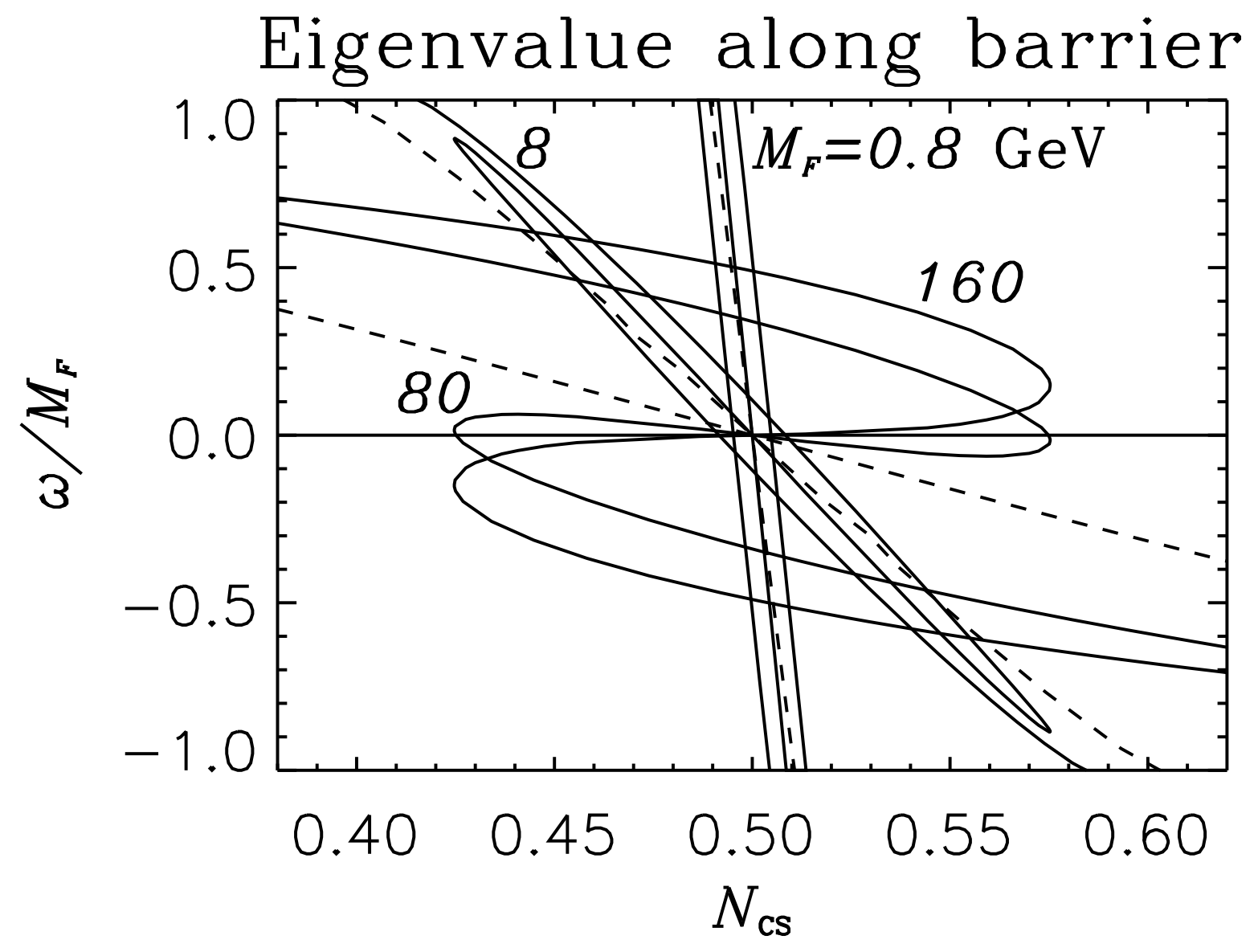




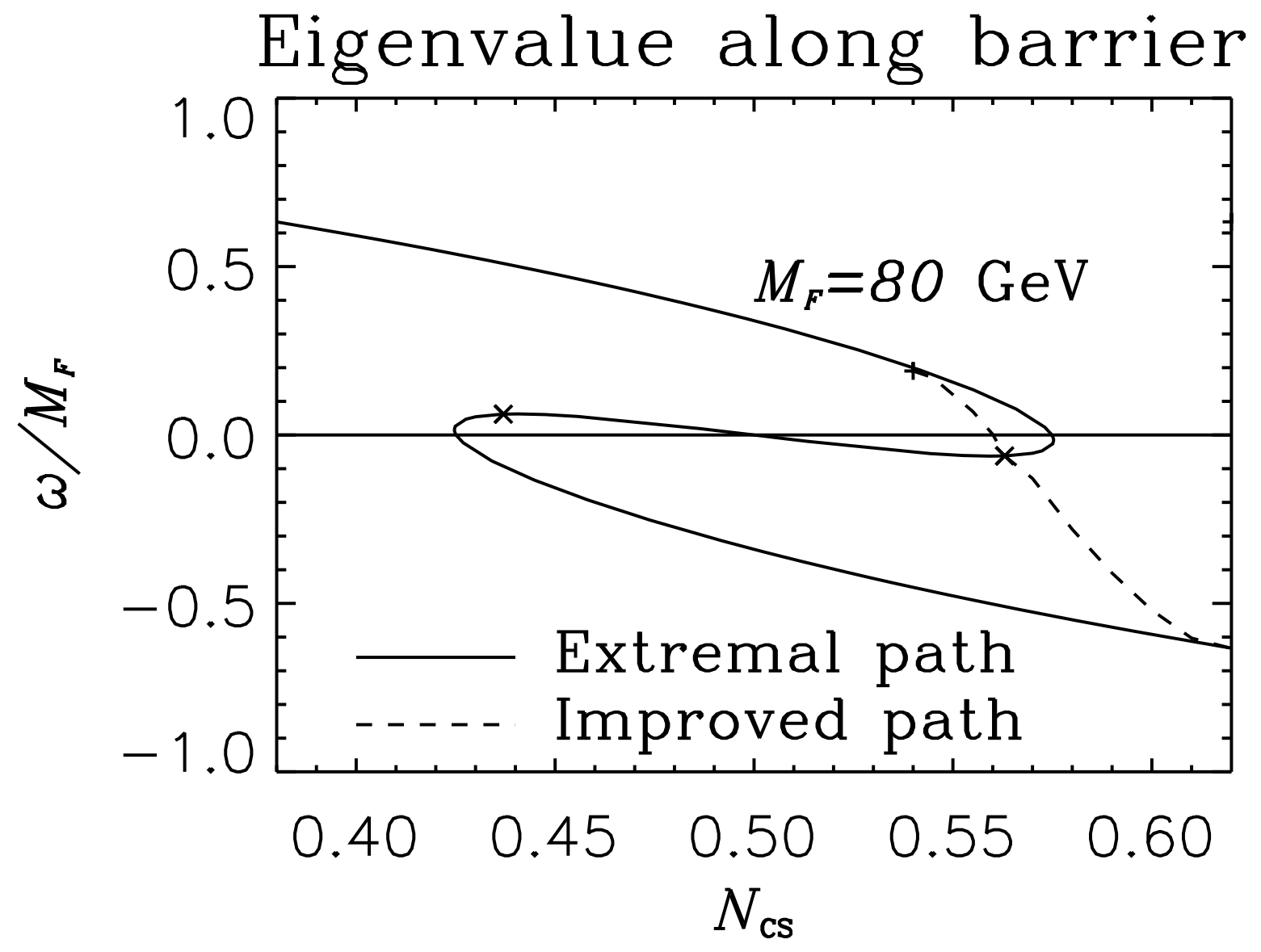




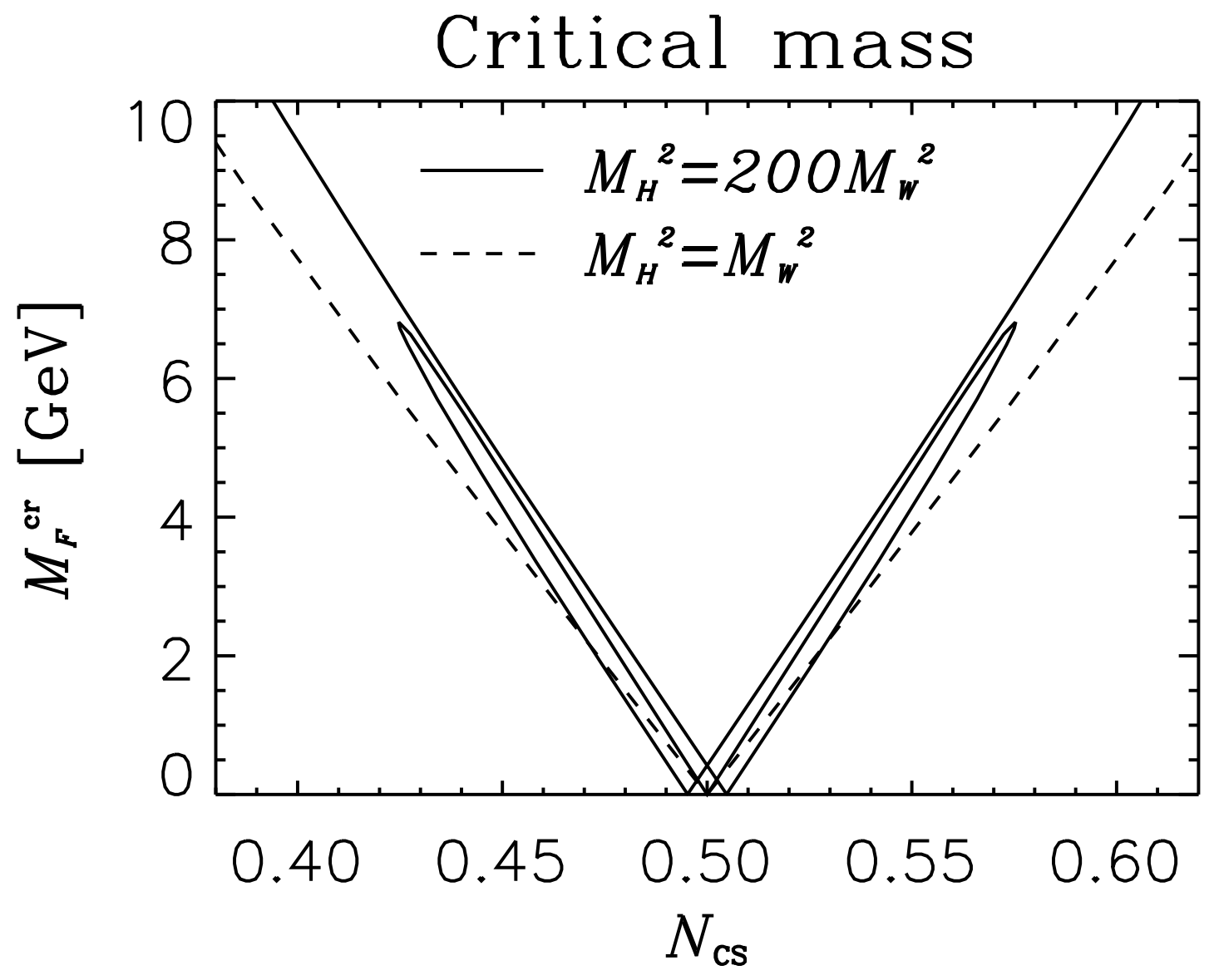




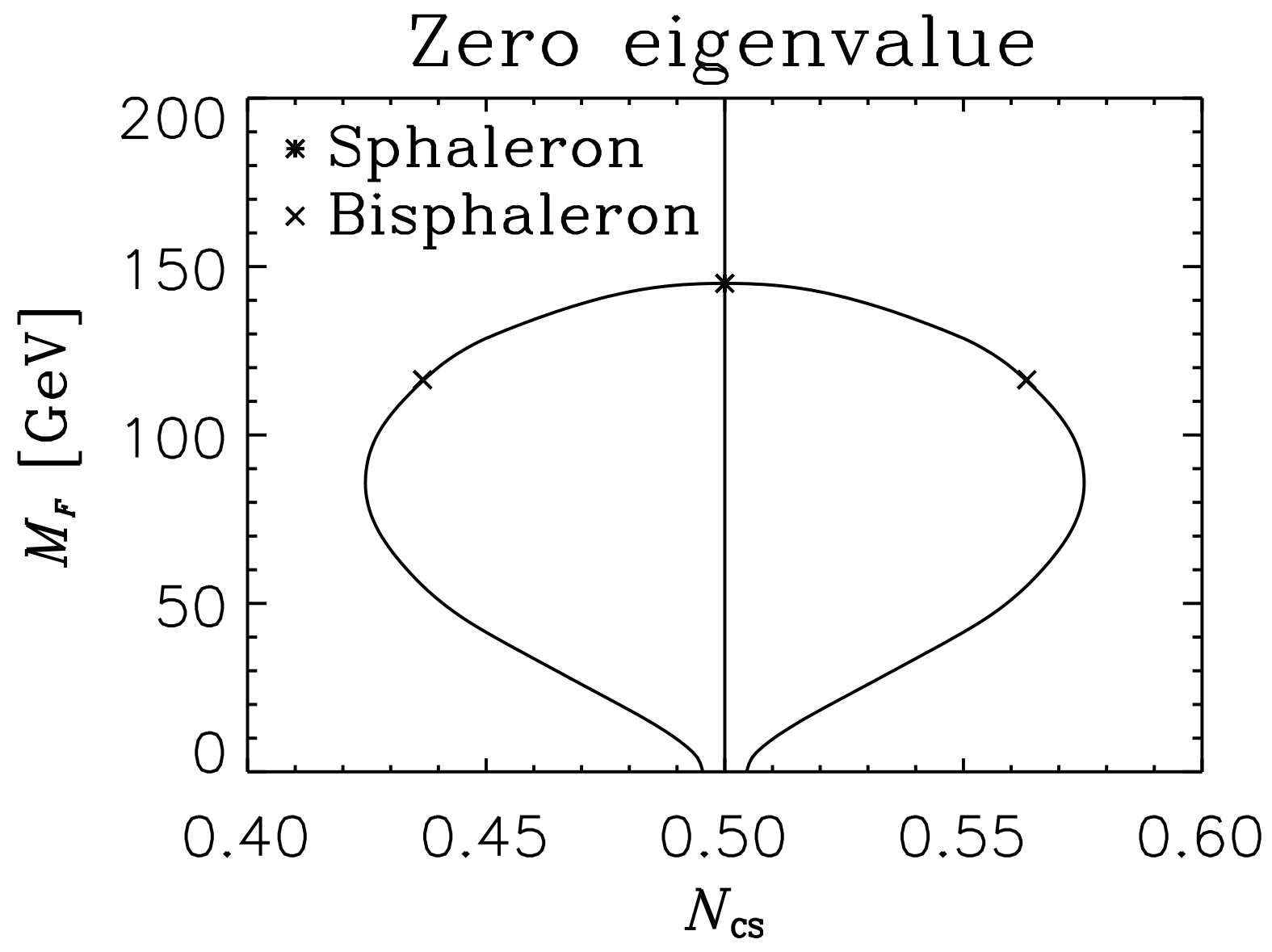

